\title{
Convex hull method for the determination of vapour-liquid equilibria (VLE) phase diagrams for binary and ternary systems.
}

\author{
Amieibibama Joseph ${ }^{\mathrm{a}, \mathrm{b}, *}$, Christine M. Sands ${ }^{\mathrm{a}}$, Peter D. Hicks ${ }^{\mathrm{a}}$, Howard W. Chandler ${ }^{\mathrm{a}}$ \\ ${ }^{a}$ School of Engineering, Fraser Noble Building, King's College, University of Aberdeen, UK. \\ ${ }^{b}$ Department of Petroleum Engineering, University of Port Harcourt, Port Harcourt, Nigeria.
}

\begin{abstract}
Flash calculations are widely used and constitute an integral part of modelling vapour-liquid equilibria in compositional simulators. However, it has been discovered that during compositional simulations, flash calculations take $50-70 \%$ of the overall computational time because the procedure currently used is iterative. Hence, several methods such as the reduced variable method, compositional space adaptive tabulation (CSAT) and the tie-line table look-up (TTL) have been developed to improve on the computational speed of most flash calculations during compositional simulations. Unfortunately, most of these methods are still iterative, and pose convergence problems, even though some are developed with efficient Newton-Raphson algorithms. Non-iterative techniques may be the best option to speed up the computational time during simulations. This paper presents a non-iterative procedure for the determination of fluid phase diagrams using the convex hull method and Peng-Robinson equation of state. Convex hull is a mathematical method, and algorithmic implementations of this method are available in many software packages, of which Matlab was used in this work. Unlike the conventional flash calculation method, programs developed with convex hull does not the need an accurate start value to make fluid phase diagrams and determine phase properties for binary and ternary mixtures. The time taken to complete a simulation run using convex hull and the conventional flash calculation method were noted, and the numerical results from both methods was validated against a range of experimental data for different mixtures. The results show good agreement in all the cases investigated. From the analyses, it was shown that the convex hull method is faster than the conventional flash calculation method in achieving convergence and also gave better predictions close to the critical point. The reliability of the results and the additional time benefits are indication that the convex hull method has a promising prospect of becoming an efficient procedure for modelling vapour-liquid phase equilibria calculations for compositional simulations.
\end{abstract}

Keywords: Convex hull, Peng-Robinson EoS, excess Gibbs energy, phase diagram, vapour-liquid equilibria, flash calculation.

\section{Introduction}

During petroleum production, the most important fluids considered are the hydrocarbon liquid and gas phases. Water also exists in every hydrocarbon bearing reservoir as an additional liquid phase but, in general, only how its flow affects hydrocarbons production is considered. These phases can coexist in equilibrium when the variables describing change in the entire system remain constant over time and position [1]. The main variables which determine the state of equilibrium in a system are the temperature, pressure and composition of that system. The ability of the vapour phase and the liquid

\footnotetext{
${ }^{*}$ Corresponding author

Email address: joseph.amieibibama@ipsng.org (Amieibibama Joseph)
} 
phase to coexist and remain in a static condition such that the number of molecules leaving and returning to a phase are equal over time and space is described as vapour-liquid phase equilibrium (VLE).

Vapour-liquid phase equilibria (VLE) calculations are used extensively in the oil and gas industry to determine the quantity (percent by volume) and quality (mole fraction of components) of the vapour and liquid phases for a given hydrocarbon composition existing at a particular temperature and pressure [2]. VLE calculations can also be used to determine phase boundaries through dew-point and bubble point calculations. With an appropriate equation of state, such information can be used to determine phase properties like density, viscosity and apparent molecular weight, which are vital for fluid flow modelling and in the production of phase diagrams.

Oil and gas reservoirs depletion processes are best described using phase diagrams. Different types of phase diagrams often used to describe the behaviour of reservoir fluids during depletion include: the pressure-temperature $(p-T)$, pressurecomposition $(p-x)$, temperature-composition $(T-x)$ and ternary phase diagrams. For two component mixtures, two coordinates phase diagrams such $(p-x),(T-x)$ or $(p-T)$ are sufficient to give adequate representation while for a three component system, a three coordinate phase diagram such as the ternary phase diagram is required. However, for a multicomponent mixture like a typical reservoir fluid, its phase composition data cannot be represented with two or three coordinates using $(p-x),(T-x)$ or ternary phase diagrams [3], but with $(p-T)$ phase diagrams. Hence, $(p-T)$ phase diagram may be considered to be the most useful in the oil and gas industry since it shows the effects of pressure and temperature on the physical state of a hydrocarbon system for any number of components during the depletion process from inception of production to abandonment [4]. Phase diagrams are very important because they give convenient representation of the ranges of temperature, pressure and composition within which different phases coexist in equilibrium [3]. Information from such diagrams plays a vital role in the design of recovery processes, pipeline transportation and separator designs [5].

More often in the oil and gas industry, VLE calculations are executed using conventional flash calculations. Flash calculations are widely employed and have remained an integral part of reservoir and chemical engineering simulations such as chemical process simulations, pipeline simulations and compositional simulations [6]. Speed is a major concern when simulating all these processes, however, research has shown that flash calculations are computationally intensive and are the most time consuming part of the simulation processes, taking about 50-70\% of the computational time, particularly in compositional simulations $[7,6]$. Flash calculations are computationally intensive because they are iterative. The Rachford-Rice [8] objective function flash calculation method, also called the conventional flash calculation is the most popular method; albeit modified versions of it have been proposed to improve on the computational time by different researchers $[9,10]$. Some other notable methods that have been proposed that do not rely on an objective function to reduce the computational time of flash calculations during compositional simulations are: the reduced variables method $[11,12,13]$; shadow-region approach $[14,15]$; the compositional-space adaptative tabulation (CSAT) method [16, 17] and the tie-line table look-up (TTL) [18] method. Unfortunately, most of these methods are also iterative, and still pose convergence problems, even though some are developed with efficient Newton-Raphson algorithms.

In this paper, a faster and non-iterative method using the convex hull algorithm has been developed to calculate the vapour-liquid phase equilibria and also to make various phase diagrams. The convex hull method uses available functions whose implementation does not require such an advanced level of computational expertise and also avoids errors reported by Baker et al. [2] such as predicting incorrect number of phases or compositions. The traditional Rachford-Rice flash calculation method is iterative and convergence to the correct solution is strongly dependent upon the initial estimate of 
the solution. If the initial estimate is insufficiently accurate, then the Rachford-Rice iterative method may not converge to the correct solution. The convex hull method is non-iterative and hence can determine the solution without being susceptible to convergence issues. The phase composition is directly determined using a proportionality rule from the Gibbs energy, instead of the iterative Rachford-Rice function in the conventional flash calculation method. The application of the convex hull method has been extended to vapour-liquid equilibria in this work, and its superiority at predicting phase compositions and phase properties close to the critical point over conventional flash calculations is illustrated. Moreover, a procedure for increasing the accuracy of $p-T$ plots, particularly close to the critical point, by refining the ternary plots is also presented and has been shown to be beneficial.

In the following sections, the convex hull method is described using the Gibbs energy and its application illustrated with the Peng-Robinson equation of state. With the Peng-Robinson equation of state and the convex hull, $(p-x),(p-T)$ and ternary phase diagrams were developed, and the results validated with experimental data and pre-existing numerical software (NIST RefProp), for different mixtures. The reduced run-time of the convex hull method is explored in greater detail and demonstrated with comparative analyses using the conventional flash calculations method.

\section{Method}

Solving vapour-liquid phase equilibria requires the application of an appropriate equation of state. In this paper, the Peng-Robinson cubic equation of state is used because of its popularity in the oil and gas industry and it is reproduced below.

\section{Peng and Robinson Equation of State}

Peng and Robinson's equation of state can be expressed in a variety of different ways. The following version is presented as Equation 4 in their classic paper [19] and relates the pressure to the molar volume $V$ in terms of parameters $a$ and $b$, which are functions of the critical temperature and pressure. Conventional notation is used, where $R$ is the universal gas constant, $T$ is the temperature and the subscript $c$ indicates the critical value. For a pure substance, $a$ and $b$ are defined as follows:

$$
a=a_{c}\left[1+\left(0.37464+1.54226 \omega-0.26992 \omega^{2}\right)\left(1-\sqrt{T_{r}}\right)\right]^{2},
$$

where $T_{r}$ is the reduced temperature $\left(T / T_{c}\right), \omega$ is the acentric factor and

$$
a_{c}=0.45724 \frac{R^{2} T_{c}^{2}}{p_{c}}
$$

and

$$
b=0.07780 \frac{R T_{c}}{p_{c}} .
$$

The Peng and Robinson Equation of State can then be defined in the form that appears as Equation 4 in their paper of 1976 as follows:

$$
p=\frac{R T}{V-b}-\frac{a(T)}{V(V+b)+b(V-b)} .
$$

Another form of this equation of state, which can sometimes be more useful, is given for one mole of fluid in terms of the gas compressibility factor, $Z$. That form appears as Equation 5 in the classic Peng-Robinson paper and is reproduced 
below:

$$
Z^{3}-(1-B) Z^{2}+\left(A-3 B^{2}-2 B\right) Z-\left(A B-B^{2}-B^{3}\right)=0
$$

where

$$
\begin{aligned}
& Z=\frac{p V}{R T}, \\
& A=\frac{a p}{R^{2} T^{2}}, \\
& B=\frac{b p}{R T} .
\end{aligned}
$$

Peng and Robinson [19] solved Equation 5 for a pure substance by utilizing the circumstance that, at the equilibrium state, the fugacity of the vapour phase $f^{v}$, equals the fugacity of the liquid phase $f^{l}$. Peng and Robinson extended their equation of state to a mixture of two components $(i$ and $j$ ) by replacing the parameters $a$ and $b$ in Equation 4 by ones obtained using conventional mixing rules and an empirically determined binary interaction coefficient, $\delta_{i j}$ :

$$
a=\sum_{i=1}^{N} \sum_{j=1}^{N} x_{i} x_{j} a_{i j}
$$

where

$$
\begin{aligned}
a_{i j} & =\left(1-\delta_{i j}\right) a_{i}^{\frac{1}{2}} a_{j}^{\frac{1}{2}}, \\
b & =\sum_{i=1}^{N} x_{i} b_{i},
\end{aligned}
$$

and $x_{i}, x_{j}$ are the mole fractions of components $i$ and $j$ in the mixture. The fugacity coefficients for the liquid and vapour phases of each component, in a mixture of $N$ components, could then be equated to obtain the required solutions.

The liquid fugacity coefficient $\Phi_{k}^{l}$, and the vapour fugacity coefficient $\Phi_{k}^{v}$, of component $k$, with mole fraction $x_{k}$ were calculated using the following equations:

$$
\begin{aligned}
& \ln \Phi_{k}^{l}=\ln \left(\frac{f_{k}^{l}}{x_{k}^{l} p}\right)=\frac{b_{k}}{b}\left(Z^{l}-1\right)-\ln \left(Z^{l}-B\right)-\frac{A}{2 \sqrt{2 B}}\left(\frac{2 \sum_{i=1}^{N} x_{i} a_{i k}}{a}-\frac{b_{k}}{b}\right) \ln \left(\frac{Z^{l}+(1+\sqrt{2}) B}{Z^{l}-(1-\sqrt{2}) B}\right), \\
& \ln \Phi_{k}^{v}=\ln \left(\frac{f_{k}^{v}}{x_{k}^{v} p}\right)=\frac{b_{k}}{b}\left(Z^{v}-1\right)-\ln \left(Z^{v}-B\right)-\frac{A}{2 \sqrt{2 B}}\left(\frac{2 \sum_{i=1}^{N} x_{i} a_{i k}}{a}-\frac{b_{k}}{b}\right) \ln \left(\frac{Z^{v}+(1+\sqrt{2}) B}{Z^{v}-(1-\sqrt{2}) B}\right) .
\end{aligned}
$$

The Peng-Robinson cubic equation of state is solved using the flash calculations and convex hull methods to calculate the vapour-liquid phase equilibria and these are described as follows.

\subsection{Conventional Flash calculation}

Processes that involves vapour-liquid equilibrium are mostly implemented using flash calculations [20]. An example of such process is that shown in Figure 1 where a feed $F$ (with composition $z_{i}$ ), is separated into its vapour $V$ (with composition $y_{i}$ ), and liquid $L$ product (with composition $x_{i}$ ). For a system of $N$ components, the material balance for 


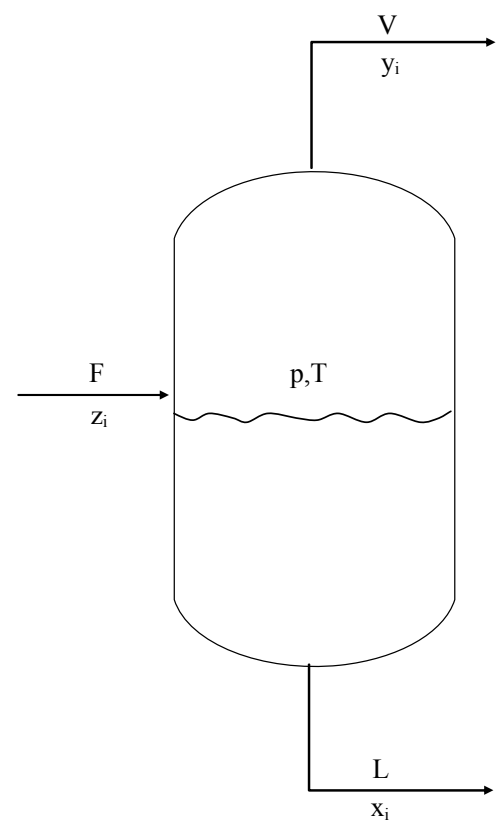

Figure 1: Schematic of a flash tank, showing the separation of a feed mixture (F) into liquid (L) and vapour (V) phases.

each component can be written as

$$
F z_{i}=L x_{i}+V y_{i} .
$$

Also, the vapour and the liquid are assumed to be in equilibrium, and hence $y_{i}=K_{i} x_{i}$, where $K_{i}$ is the equilibrium ratio. Consequently the equilibrium ratio

$$
K_{i}=\frac{y_{i}}{x_{i}},
$$

which defines the ratio of the composition of $i$ in the vapour phase to the composition of component $i$ in the liquid phase. For the $i_{t h}$ component, there will be $l_{i}$ moles present in the liquid phase and $v_{i}$ moles in the vapour phase such that: $l_{i}+v_{i}=n_{i}$. Let

$$
\begin{aligned}
x_{i} & =l_{i} / L \text { and } y_{i}=v_{i} / V, \\
z_{i} & =n_{i} / F \text { and } \sum z_{i}=1.0, \\
n_{v} & =V / F \text { and } n_{l}=L / F, \\
L & =F-V .
\end{aligned}
$$

Substituting Equation 11 into Equation 10, and also applying the relationships in Equation 12, the following expressions were derived for estimating the composition of component $i$ in the vapour and liquid phases:

$$
\begin{aligned}
y_{i} & =\frac{z_{i} K_{i}}{1+n_{v}\left(K_{i}-1\right)}, \quad \text { where } \quad \sum_{1=1}^{N} \frac{z_{i} K_{i}}{1+n_{v}\left(K_{i}-1\right)}=1, \text { for the vapour phase; } \\
\text { and } x_{i} & =\frac{z_{i}}{1+n_{v}\left(K_{i}-1\right)}, \quad \text { where } \quad \sum_{1=1}^{N} \frac{z_{i}}{1+n_{v}\left(K_{i}-1\right)}=1 \text {, for the liquid phase. }
\end{aligned}
$$



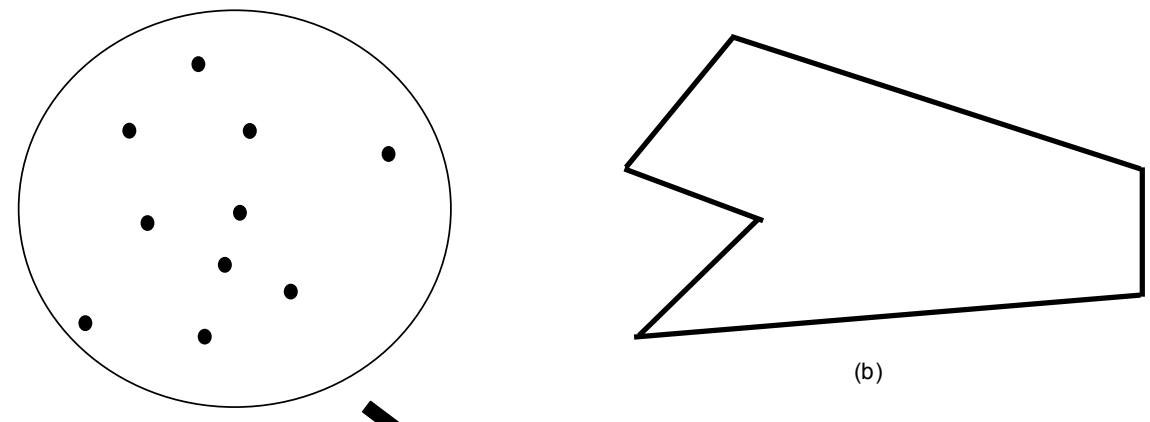

(b)

(a)

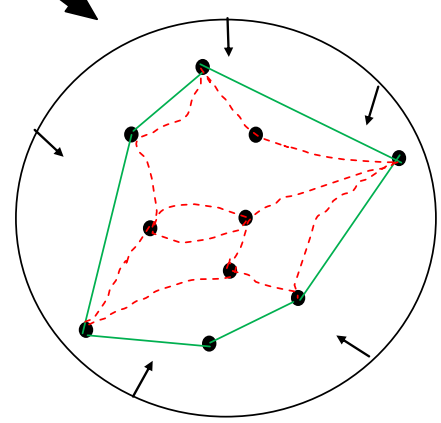

(c)

Figure 2: The making of a convex hull, (a) a typical convex shape, (b) a non-convex shape, and (c) showing outlines that define a convex from Figure 2a.

Combining Equations (13) and (14) i.e. $\sum_{i=1}^{N}\left(y_{i}-x_{i}\right)=0$ results in an equation with good numerical properties, and this is called Rachford and Rice [8] objective function given as:

$$
F\left(n_{v}\right)=\sum_{1=1}^{N} \frac{z_{i}\left(K_{i}-1\right)}{1+n_{v}\left(K_{i}-1\right)}=0
$$

The conventional flash calculations were performed in this work by a modified version of the code of Affrough [21] that uses Equation 15.

\subsection{Convex hull}

A body is said to be convex if a tangent drawn on any part of the surface never enters the body interior, or if any straight line drawn through the body intersects its boundaries exactly twice. In mathematics, a convex hull $(S)$ of a set of points, is the smallest convex set that contains the points [22,23], and the convex combination of the points in the set can be expressed as:

$$
S \equiv \sum_{j=1}^{N} \lambda_{j} p_{j} \quad \text { where: } \lambda_{j} \geq 0 \quad \text { for all } \quad j \quad \text { and } \quad \sum_{j=1}^{N} \lambda_{j}=1 .
$$

Here, $N$ is the total number of points, $p_{j}$ is a vector that represent the points that make up the set while $\lambda_{j}$ is a scalar that represent the coefficient to each point in the set. In two dimensions, a circle is convex as shown in Figure 2(a); but the hexagonal polygon shown in Figure 2(b) is not convex because a line drawn through it may intersect its boundaries more than twice. The 'hull', or outline, drawn around a set of points may, or may not be a convex. Consider the black dots shown in Figure 2(c). An outline drawn round these points (see red dashed line) would not be convex. However, the solid green polygon, is convex and encloses all the points and hence forms a convex hull.

The concept of a convex hull was originally a fundamental construction for mathematics and computational geometry 
used for the analysis of spectrometry data, canonical triangulation, Delaunay triangulation, Voronoi diagrams and halfspace intersection $[24,25,22]$. Convex hulls have wide application in metallurgy, urban planning, crystallography, collision detection, statistics, point location, numerical integration and cartography [22]. Recently, the application of convex hull has been extended to the chemical industry in the determination of phase diagrams for ternary and quaternary systems using the convex envelope method (CEM) [26] and the interpretation of Gibbs tangent plane of phase equilibria of multicomponent mixtures [23]. However, fluid phase equilibria convex hull have so far been applied to the excess Gibbs energy $\left(g^{E}\right)$ models to describe liquid-liquid phase equilibria using the activity coefficient. In the oil and gas industry, vapourliquid phase equilibria are of more interest than liquid-liquid phase equilibria because virtually all hydrocarbon systems involve the coexistence of liquid and vapour phases. In this paper, the application of convex hull has been extended to vapour-liquid phase equilibria using the Peng-Robinson equation of state. The convex hull is a mathematical method, and the sources of the algorithm are available in many software packages, of which Matlab was used in this paper. In algorithms that construct convex hulls, the points that make up a set are first sorted, and then the upper and lower hulls are constructed. By specifying the system variables (in this case pressure, temperature and feed composition), and stepping through a range of points, the Gibbs energy at each point is obtained from the fugacity expressions. A convex hull can then be constructed and utilized to determine a phase equilibria. For instance, in developing the $p-x$ phase equilibrium predictions for a carbon-dioxide-isobutane mixture, 250 points are sufficient. However, for other mixtures, additional points may be necessary, although this is dependent on the number of compositions and the system conditions. In this work, a two dimensional implementation of the convex hull algorithm was used. However, higher dimensional variants of the algorithm are also available.

\subsection{Gibbs energy and convex hull analysis}

A typical hydrocarbon system consists of a range of light to heavier components. One convenient way of considering a mixture of more than two components is to work in terms of the partial molar Gibbs energy. The partial molar Gibbs energy (also called the chemical potential), is commonly denoted as $\mu_{k}$ for component $k$ and may be defined for each phase as:

$$
\mu_{k}=\left(\frac{\partial G}{\partial n_{k}}\right)_{p, T, n}
$$

where $n_{k}$ is the number of moles of component $k$ in a mixture of $N$ components (or species), and $n$ is the number of moles of other components in the mixture. For each component in a two phase mixture at equilibrium, the chemical potential in the liquid phase will be the same as that in the vapour phase. Hence, the total Gibbs energy for a mixture can also be defined as:

$$
G=\sum_{k=1}^{N}\left(\mu_{k}^{l} n_{k}^{l}+\mu_{k}^{v} n_{k}^{v}\right) .
$$

The mole fraction of species $k$ is $x_{k}=n_{k} / n_{t}$, where $n_{t}$ is the total number of moles. The partial molar Gibbs can also be defined in terms of the fugacity $\left(f_{k}^{i}\right)$ of species $k$ as:

$$
\begin{aligned}
& \mu_{k}^{l}=\mu_{k}^{\Theta}+\left(\ln f_{k}^{l}-\ln p^{\Theta}\right), \\
& \mu_{k}^{v}=\mu_{k}^{\Theta}+\left(\ln f_{k}^{v}-\ln p^{\Theta}\right),
\end{aligned}
$$

where superscript $\Theta$ represents the chemical potential at a reference state; usually denoting the value of the chemical potential at the start of an investigation. 


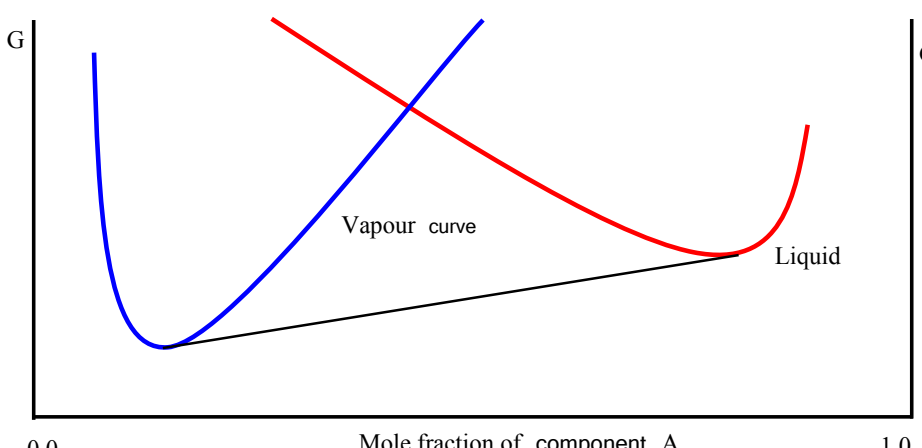

0.0
Mole fraction of component $\mathrm{A}$

(a)

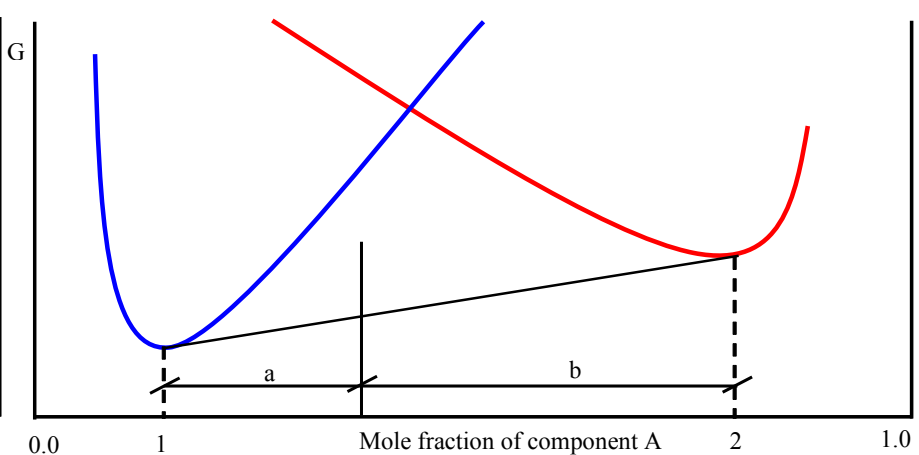

(b)

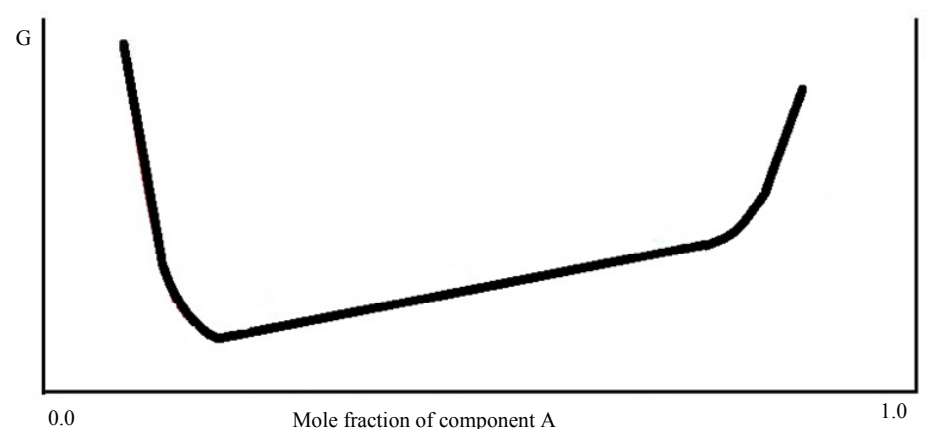

(c)

Figure 3: (a) Schematic of change in Gibbs' energy versus composition for a two component mixture. Vapour Gibbs energy shown as a thick blue line, the liquid Gibbs energy as a thick red line and the common tangent shown as a thin black line. (b) Interpretation of schematic shown in Figure 3(a). (c) Convex hull for Figure 3(a).

Figure 3(a) shows a schematic plot of Gibbs' energy versus mole fraction for a notional two component (or two species) mixture, at an arbitrary combination of pressure and temperature, as a vapour (shown as a thick red line) and a liquid (shown as a thick blue line). As Gibbs' energy can only be expressed relative to some arbitrary reference, this strictly only illustrates the change in the Gibbs' energy with composition for this particular combination of pressure and temperature. Figure 3(b) illustrates how this may be interpreted. For the particular composition indicated by the vertical black solid line, the mixture will consist of a mole fraction of vapour, $x_{v}=b /(a+b)$, with the composition indicated by position 1 ; and a mole fraction of liquid, $x_{l}=a /(a+b)$, of the composition indicated by position 2 , as this combination produces the lowest value of Gibbs' energy. Compositions to the left of position 1 can only exist as a vapour, at this particular temperature and pressure, while to the right of position 2 they can only exist as a liquid. Figure 3(c) shows, by the thick black line, the curve that defines this. It should be noted that the shape of this curve is convex, and no tangent drawn to the curve will cross the curve at any other point. This feature permits the use of convex analysis. The thick black line shown in Figure 3(c) is the convex hull. At equilibrium, the fugacity of the liquid phase is equal to that in the vapour phase. The slope of the thin black line shown in Figure 4 is the same where it touches the blue curve and the green curve. That is equivalent to the fugacities being the same in both phases [27]. The algorithm for implementing the convex hull analysis can found in Barber et al. [22].

\subsection{Illustrating Convex hull with the Equation of State of Peng and Robinson}

The Peng-Robinson equation of state is one of the most commonly used cubic equations of state in the oil and gas industry due to its superiority in estimating the density over other known cubic equations of state [28]. It has also shown reasonable accuracy near the critical point, particularly in the calculation of the gas compressibility factor [29]. These 


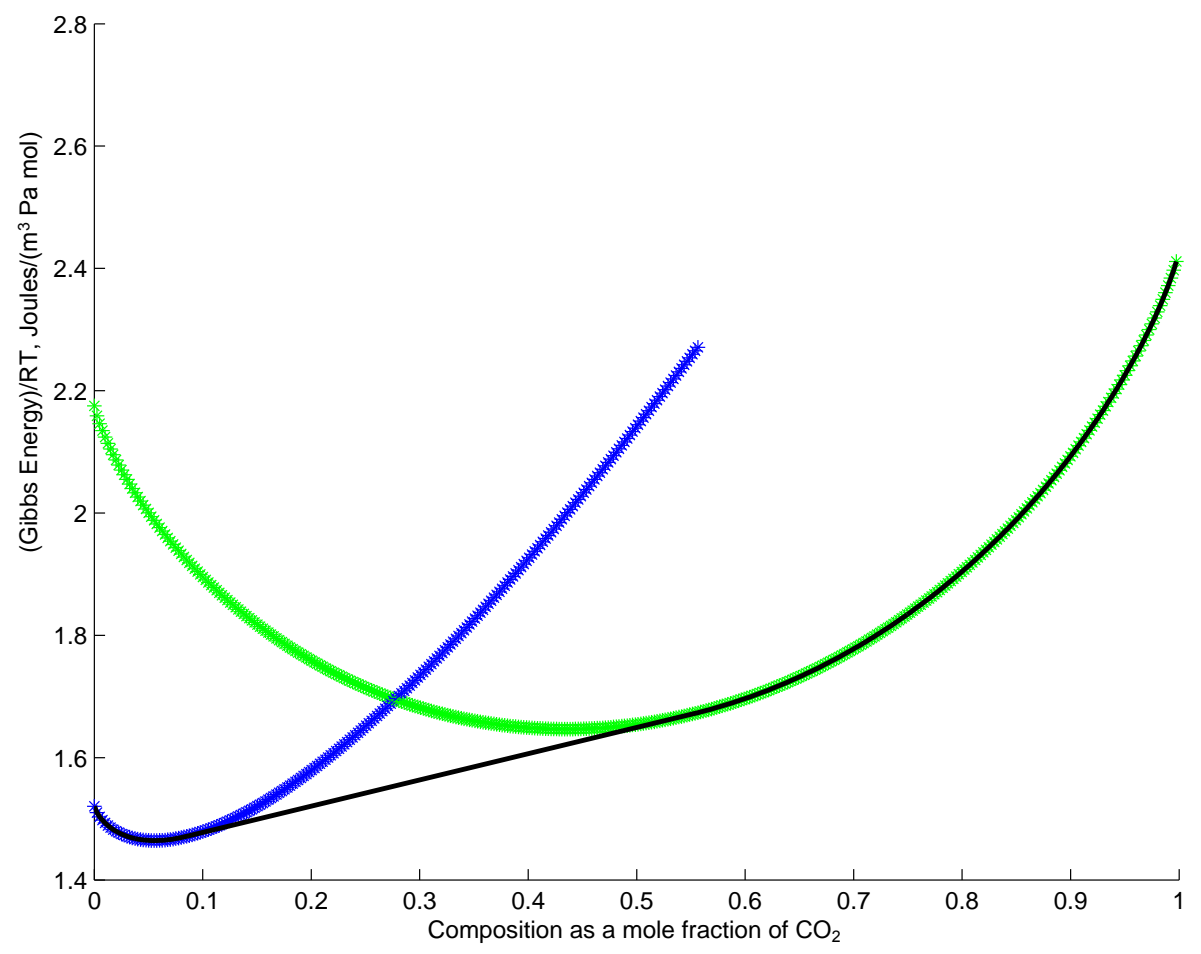

Figure 4: Plot of change in Gibbs energy versus composition for a two component mixture of carbon-dioxide and isobutane. vapour plot shown as a thick green line, the liquid plot as a darker thick blue line and the hull shown as a thin black line.

parameters are needed when simulating fluid flow dynamics and understanding the behavior of fluid in hydrocarbon reservoirs [30].

Although the Peng-Robinson equation of state, being a relatively simple two parameter cubic equation of state, is quite easy to solve, using it to determine the parameters needed to simulate fluid flow usually requires an intensive iterative procedures that in many cases gives convergence problems. Some of these problems can be difficult to overcome as they are strongly dependent on accurate start values for the iterative procedure.

\section{Results and Discussions}

Figure 4 shows a plot of Gibbs energy divided by $R T$, versus mole fraction for a carbon-dioxide and isobutane mixture at an arbitrarily chosen pressure and temperature of $5.165 \mathrm{MPa}$ and $370 \mathrm{~K}$; with vapour (as a green line) and liquid (as a darker blue line). Convexity algorithms are appropriate for use with the mole fraction or with the number of moles. Justification for this can be found in Müller and Müller [27]. For a range of compositions at the temperature and pressure to which the plots relate, the mixture will exist as two phases, each with different compositions. The compositions of the phases are set by the ends of the straight portions of the 'hull' curve (shown as the thin black line); while the proportions of each phase are set by the composition of the mixture and proportionality rules. For example, if the straight-line portion of the liquid curve ends at mole fraction $x_{l}$, the convex portion of the vapour curve ends at mole fraction $x_{v}$, and the mixture composition at which they coexist in equilibrium is $x_{m}$, then the fraction of the total moles of the mixture composition $x_{m}$, in the vapour phase will be $\left(x_{m}-x_{l}\right) /\left(x_{v}-x_{l}\right)$, while the number of moles in the liquid phase will be $\left(x_{v}-x_{m}\right) /\left(x_{v}-x_{l}\right)$. The values of $x_{l}$ and $x_{v}$ can easily be obtained using the convex hull by the procedure illustrated with Figure 3(b).

The convex hull method was first validated and compared with results from flash calculations method using data 


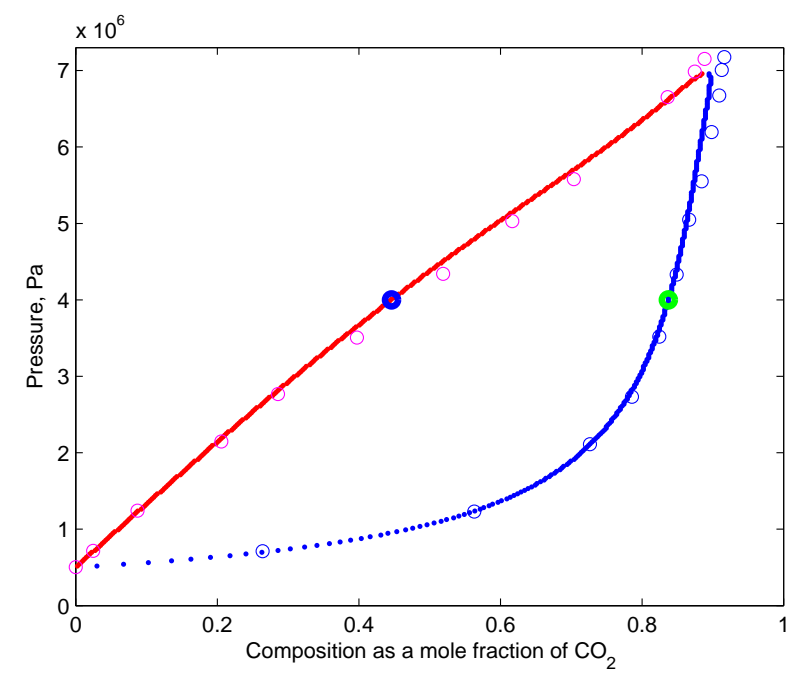

(a)

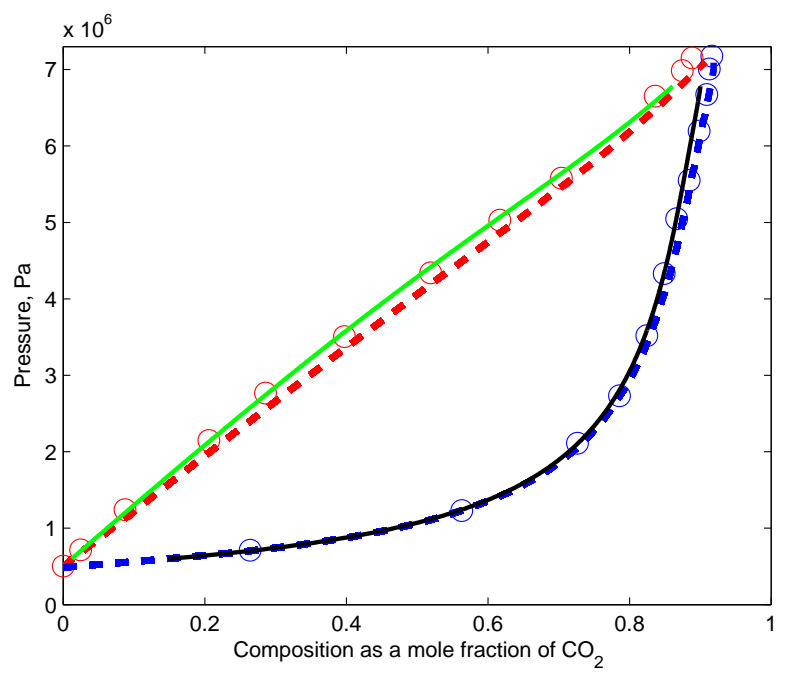

(b)

Figure 5: Pressure versus composition for carbon-dioxide-isobutane mixture at 310.9K. (a) Showing how relevant data can be obtained easily, e.g the composition at $4 \mathrm{MPa}$ (and $310.9 \mathrm{~K}$ ) of the liquid phase (thick blue circle is 0.4914 ) and the vapour phase (thick green circle is 0.8468 ) were determined directly without the need to iterate from the start value, illustrating the suitability of the approach to simulate flash calculations. (b) Comparing results from convex hull with conventional flash calculations and experimental data. Results from convex hull are shown as red and blue-dash lines for the bubble and dew point lines while that from flash calculation as solid green and black lines. The red and blue circles are data obtained from Peng-Robinson paper [19].

provided by Peng and Robinson [19] for a mixture of carbon-dioxide and isobutane as shown in Figure 5. Further validation was then completed for a range of temperatures for a methane-npentane mixture from NSRDS-NBS 32 data [31] as shown in Figure 6 and for methane-nbutane mixtures using data from Elliot et al [32] as shown in Figure 7. Figures 5 to 7 shows the excellent performance of this procedure by accurately matching these experimental data; a demonstration of the reliability of the convex hull method. Moreover, the convex hull method also gave better predictions of fluid properties near the critical point than the conventional flash calculation method as results from convex hull are closer to the experimental data than flash calculation. However, Figure 7 shows obvious disparity in the values of the critical loci obtain from the experimental data with that obtained using either the flash calculation method or the convex hull. Elliot et al. [32] also reported that the critical loci determined in their work show significant deviations in the low temperature range compared to what is widely used in the industry. Therefore, the result from convex hull and the flash calculation methods at the critical loci might be more reliable than the experimental values.

Obviously, other procedures exist $[33,29]$ that would produce the curves shown in Figures 5 to 7 , but the programs may take much a longer time to run and usually rely on accurate start values and a small increment to produce satisfactory results. The authors tested this using conventional optimization procedures with the Peng-Robinson model to produce the same curves shown in Figures 5 to 7 . Additionally, although it is relatively simple to produce full curves starting with accurate data for the origin, but producing results for pressures remote from the origin directly (i.e. without stepping from the origin to the relevant pressure) is more difficult. The approach presented in this paper also enables relevant data to be obtained directly and more quickly (the blue and green circles shown on Figure 5(a)); taking less than $1.0 \%$ of the time taken (using the convex hull approach) to determine any data point using the full run. Clearly the time benefit will vary depending on the pressure chosen and the approach used to solve the Peng-Robinson equation.

For each simulation, the time taken to generate a phase diagram and its associated phase properties were recorded as 


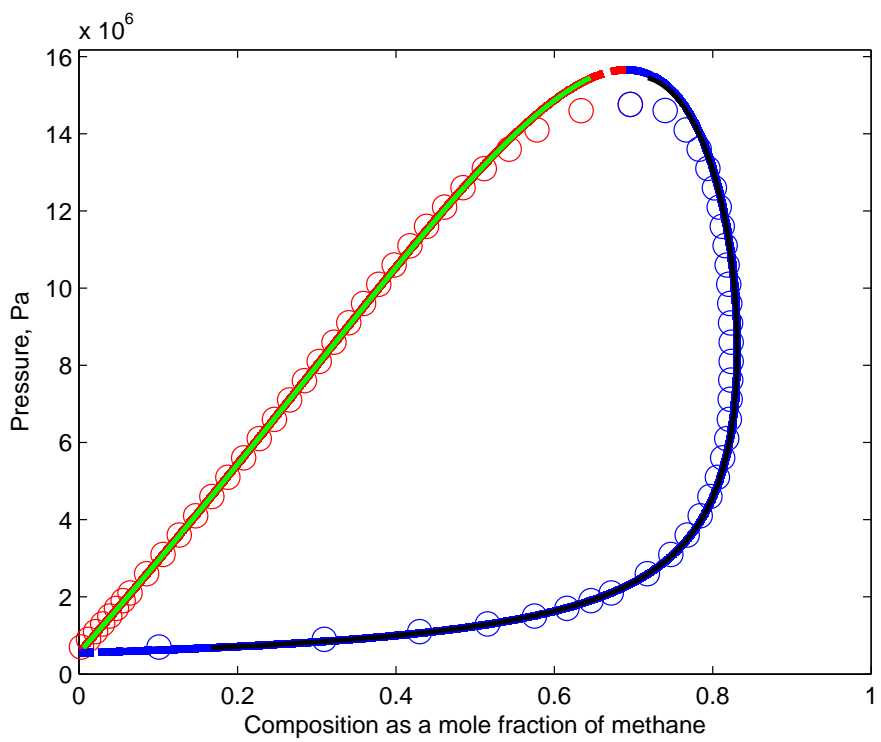

Figure 6: Comparison of pressure-compositions for $\mathrm{C}_{1}-\mathrm{nC}_{5}$ mixture at $370 \mathrm{~K}$ using convex hull and flash calculation: Results from convex hull are shown as red and blue-dash lines for the bubble and dew point lines while that from flash calculation as green and black lines. The red and blue circles are data obtained from Berry and Sage [31].

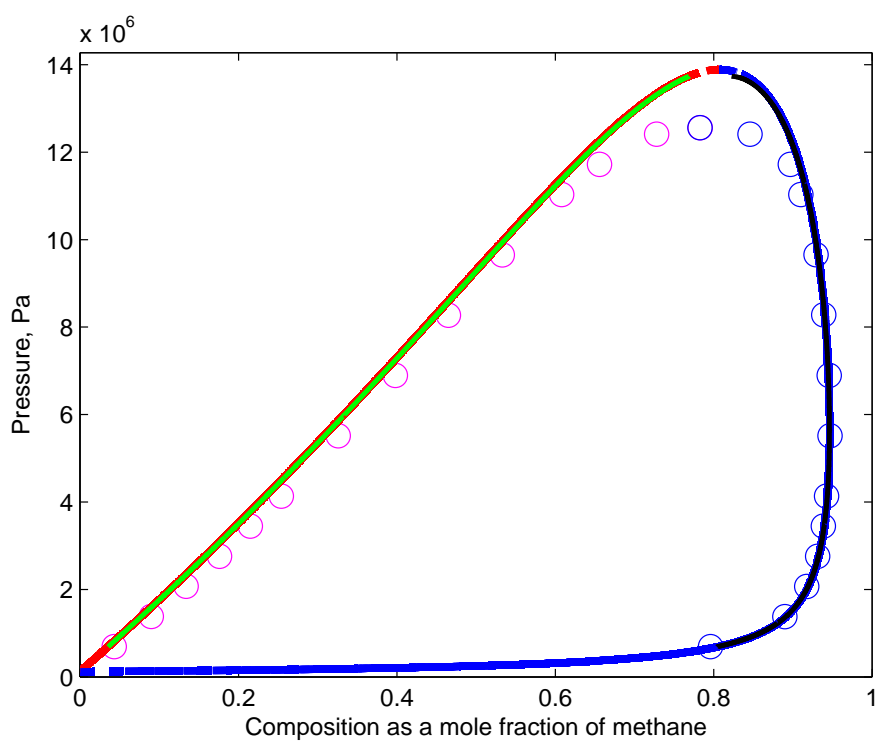

Figure 7: Comparison of pressure-compositions for $\mathrm{C}_{1}-\mathrm{nC}_{4}$ mixture at $277.59 \mathrm{~K}$ using convex hull and flash calculation: Results from convex hull are shown as red and blue-dash lines for the bubble and dew point lines while that from flash calculation as green and black lines. The red and blue circles are data obtained from Elliot et al. [32]. 


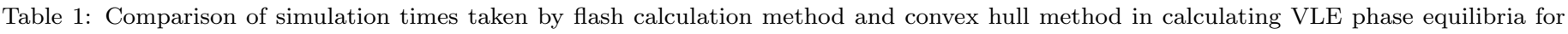
different mixtures.

\begin{tabular}{|lll|}
\hline Phase diagram type & Convex hull & Flash calculation \\
\hline Time & seconds & seconds \\
$p-x$ for carbon-dioxide-isobutane mixture & 0.323 & 3.309 \\
$p-x$ for methane-pentane mixture & 0.222 & 12.250 \\
$p-x$ for methane-nbutane mixture & 0.624 & 100.063 \\
\hline
\end{tabular}

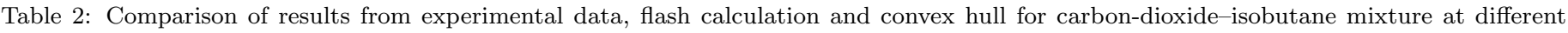
pressures and temperatures

\begin{tabular}{|c|c|c|c|c|c|c|c|c|}
\hline Properties & \multicolumn{2}{|l|}{ Expt. } & \multicolumn{2}{|l|}{ NIST } & \multicolumn{2}{|c|}{ Flash cal. } & \multicolumn{2}{|c|}{ Convex hull } \\
\hline $\begin{array}{l}\text { Components } \\
\text { @ } 5.054 \mathrm{MPa} \text { and } 310.9 \mathrm{~K}\end{array}$ & $\mathrm{CO}_{2}$ & $\mathrm{iC}_{4}$ & $\mathrm{CO}_{2}$ & $\mathrm{iC}_{4}$ & $\mathrm{CO}_{2}$ & $\mathrm{iC}_{4}$ & $\mathrm{CO}_{2}$ & $\mathrm{iC}_{4}$ \\
\hline Vapour mole fraction, $y_{i}$ & 0.8690 & 0.1310 & 0.8708 & 0.1292 & 0.8663 & 0.1337 & 0.8759 & 0.1241 \\
\hline liquid mole fraction, $x_{i}$ & 0.6205 & 0.3795 & 0.6278 & 0.3722 & 0.6146 & 0.3854 & 0.6456 & 0.3544 \\
\hline Vapour compressibility, $Z_{v}$ & 0.6556 & & 0.6174 & & 0.6148 & & 0.6162 & \\
\hline Liquid compressibility, $Z_{l}$ & 0.1619 & & 0.1748 & & 0.1710 & & 0.1676 & \\
\hline Vapour density, $\rho_{g}$ & 136.76 & & 145.790 & & 146.188 & & 145.188 & \\
\hline Liquid density, $\rho_{l}$ & 596.17 & & 551.048 & & 565.857 & & 571.568 & \\
\hline @ $2.13 \mathrm{MPa}$ and $310.9 \mathrm{~K}$ & & & & & & & & \\
\hline Vapour mole fraction, $y_{i}$ & 0.7297 & 0.2703 & 0.7401 & 0.2599 & 0.7293 & 0.2707 & 0.7347 & 0.2653 \\
\hline liquid mole fraction, $x_{i}$ & 0.2056 & 0.7944 & 0.2179 & 0.7821 & 0.2058 & 0.7942 & 0.2262 & 0.7738 \\
\hline Vapour compressibility, $Z_{v}$ & 0.8511 & & 0.8212 & & 0.8194 & & 0.8198 & \\
\hline Liquid compressibility, $Z_{l}$ & 0.0817 & & 0.0821 & & 0.0794 & & 0.0786 & \\
\hline Vapour density, $\rho_{g}$ & 46.303 & & 47.9800 & & 48.1730 & & 48.1260 & \\
\hline Liquid density, $\rho_{l}$ & 556.94 & & 554.122 & & 573.420 & & 573.868 & \\
\hline
\end{tabular}

${ }^{c}$ calculated from parameters given in Basserer and Robinson [34] .

shown in Table 1. Table 1 shows that the convex hull method is faster than the conventional flash calculation method in achieving quick convergence in the cases investigated. For example, the convex hull took less than $1 \%$ of the time taken by the flash calculation to achieve convergence for the methane-nbutane mixture and less than $10 \%$ of the time for the other cases.

In order to carry out a quantitative assessment of how each method closely predicts the experimental data, fluid properties were estimated at specific pressures and temperatures for the different mixtures. Tables 2 to 5 show the results for the phase properties of each mixture obtained from the simulation runs for the flash calculation and the convex hull. These were compared with experimental data. The phase compressibility factors and densities were not reported in these data. However, for the carbon-dioxide-isobutane and methane-npenatne mixtures, the molar volumes were reported, hence the compressibility factors are calculated using Equation 6a while the densities were calculated using

$$
\begin{aligned}
\rho_{v} & =\frac{p M_{v}}{Z_{v} R T}, \quad \text { for vapour phase; } \\
\text { and } \quad \rho_{l} & =\frac{p M_{l}}{Z_{l} R T}, \quad \text { for liquid phase. }
\end{aligned}
$$

Here $\rho$ is the density, $M$ is the apparent molecular weight and subscripts $v$ and $l$ represent the vapour and liquid phases. For the methane-nbutane mixture, only the phase compositions were reported by Elliot et al. [32], hence Peng-Robinson [19] EOS was used to estimate the compressibility factors and the phase densities. To appreciate how each method closely predicts the experimental data, an absolute and relative error analysis was also carried out to investigate the magnitude of the departures of the simulated results from the experimental data.

Table 6 shows the phase compositional departures between the experimental data and flash calculation, and between 


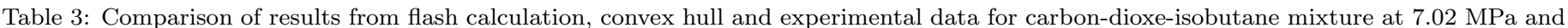
$310.9 \mathrm{~K}$

\begin{tabular}{|lllllll|}
\hline Properties & Expt. & \multicolumn{2}{l|}{ Flash cal. } & \multicolumn{2}{l|}{ Convex hull } \\
Components & $\mathrm{CO}_{2}$ & $\mathrm{iC}_{4}$ & $\mathrm{CO}_{2}$ & $\mathrm{iC}_{4}$ & $\mathrm{CO}_{2}$ & $\mathrm{iC}_{4}$ \\
Vapour mole fraction, $y_{i}$ & 0.9125 & 0.0875 & 0.8384 & 0.1616 & 0.9189 & 0.0811 \\
Liquid mole fraction, $x_{i}$ & 0.8694 & 0.1306 & 0.8384 & 0.1616 & 0.8989 & 0.1011 \\
\hline Gas compressibility, $Z_{v}$ & $0.4327^{c}$ & 0.2455 & & 0.3837 & \\
Liquid compressibility, $Z_{l}$ & $0.2285^{c}$ & 0.2455 & 0.2873 & \\
\hline Gas density, $\rho_{g}$ & $283.979^{c}$ & 512.833 & 319.573 \\
Liquid density, $\rho_{l}$ & $544.986^{c}$ & 512.833 & 429.541 \\
\hline
\end{tabular}

${ }^{c}$ are calculated from parameters given in Basserer and Robinson [34] .

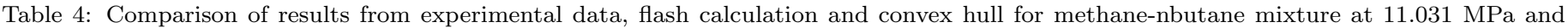
$277.59 \mathrm{~K}$

\begin{tabular}{|c|c|c|c|}
\hline Properties & Expt. & Flash cal. & Convex hull \\
\hline Components & $\mathrm{CH}_{4}$ & $\mathrm{CH}_{4}$ & $\mathrm{CH}_{4}$ \\
\hline Vapour mole fraction, $y_{i}$ & 0.9100 & 0.9179 & 0.9190 \\
\hline Liquid mole fraction, $x_{i}$ & 0.6078 & 0.5895 & 0.5886 \\
\hline Gas compressibility, $Z_{v}$ & $0.6580^{a}$ & 0.6413 & 0.6434 \\
\hline Liquid compressibility, $Z_{l}$ & $0.3771^{a}$ & 0.3595 & 0.3592 \\
\hline Gas density, $\rho_{g}$ & $144.0425^{a}$ & 145.3057 & 144.5260 \\
\hline Liquid density, $\rho_{l}$ & $412.5319^{a}$ & 442.9014 & 443.7790 \\
\hline
\end{tabular}

${ }^{a}$ estimated using Peng and Robinson [19] EOS .

Table 5: Comparison of results from experimental data, flash calculation and convex hull for methane-npentane mixture at 12.667 MPa and $370 \mathrm{~K}$

\begin{tabular}{|lllllll|}
\hline Properties & Expt. & \multicolumn{3}{l}{ Flash cal. } & \multicolumn{2}{l|}{ Convex hull } \\
Components & $\mathrm{CH}_{4}$ & $\mathrm{nC}_{5}$ & $\mathrm{CH}_{4}$ & $\mathrm{nC}_{5}$ & $\mathrm{CH}_{4}$ & $\mathrm{nC}_{5}$ \\
Vapour mole fraction, $y_{i}$ & 0.8023 & 0.1977 & 0.8057 & 0.1943 & 0.8068 & 0.1932 \\
Liquid mole fraction, $x_{i}$ & 0.4849 & 0.5151 & 0.4885 & 0.5115 & 0.4875 & 0.5125 \\
\hline Gas compressibility, $Z_{v}$ & $0.7559^{b}$ & 0.7468 & & 0.7486 \\
Liquid compressibility, $Z_{l}$ & $0.4726^{b}$ & 0.4708 & 0.4706 \\
\hline Gas density, $\rho_{g}$ & $147.8199^{b}$ & 148.5766 & 147.8823 \\
Liquid density, $\rho_{l}$ & $391.5951^{b}$ & 391.2862 & 391.9205 \\
\hline
\end{tabular}

${ }^{b}$ calculated from parameters given in Berry and Sage [31] . 
the experimental data and convex hull for all the mixtures. For the carbon-dioxide-isobutane mixtures in Table 6 , both the flash calculation and the convex hull methods closely predicted the measured compositions with very small margins of absolute and relative errors. At $5.054 \mathrm{MPa}$ and $310.9 \mathrm{~K}$, the maximum absolute error was found in the prediction of the vapour and liquid mole fractions for carbon-dioxide between the flash calculation and convex hull $( \pm 0.0096)$, while it is $( \pm 0.0310)$ for isobutane. Similarly, the maximum relative error for the the same mixture was found to occur in the prediction of the vapour mole fraction of isobutane between flash calculation and convex hull (7.180\%). For the same mixture at $2.13 \mathrm{MPa}$ and $310.9 \mathrm{~K}$, similar trends were observed and the results are consistent with the experimental data. However, it was observed that at pressures close to the critical pressure of the carbon-dioxide-isobutane mixture (8.01 $\mathrm{MPa}$ ), the predictive capability of the convex hull is much better than the flash calculation. This can be seen in Table 3, where at $7.02 \mathrm{MPa}$ and $310.9 \mathrm{~K}$, the flash calculation method gave indifferent values for the vapour and liquid phase properties estimated, which are far from the experimental results compared to the estimated values obtained from the convex hull particularly in respect of the densities. This can also be seen in the values of the absolute error calculated. With the flash calculation method, the estimated absolute errors are $( \pm 0.0741)$ and $( \pm 0.031)$ for the vapour and liquid mole fractions whereas the absolute errors for the same components were found to be $( \pm 0.0064)$ and $( \pm 0.0295)$ using the convex hull. This is also evident in the phase diagrams shown in Figures 5(b), 6 and 7 where the phase envelops does not close-in for the bubble and dew point lines. Although, the convex hull method was able to predict the properties at this condition and as well show good separation between the vapour and liquid phase properties estimated for each component, the flash calculation method is better in closely predicting the experimental results at very low pressures.

Similar analysis was also carried out for the binary mixtures of methane-nbutane and methane-npentane shown in Table 6. For these mixtures, both the flash calculation and the convex hull also closely predicted the experimental results with very small margins of absolute and relative errors. When the results from the flash calculation and convex hull were compared, an absolute error of \pm 0.0011 could be seen as shown in column four of Table 6 recurring for the prediction of the vapour and liquid mole fractions for methane-nbutane and methane-npentane mixtures. This consistency is a confirmation of the reliability of the convex hull method.

Table 7 shows the compressibility factor and density departures of the phases between the experimental data and flash calculation, and between the experimental data and convex hull respectively.

It can seen from Table 7 that both methods closely predicted the experimental data. The minimum absolute error was found to occur in the prediction of the liquid compressibility factor for methane-npentane mixture (0.0018) while the maximum absolute error occurred in the prediction of the liquid density of methane-nbutane mixture $\left(31.2491 \mathrm{~kg} / \mathrm{m}^{3}\right)$. On the other hand, the minimum relative error was found to occur in the vapour density prediction of methane-npentane mixture $(0.0422 \%)$ while the maximum relative error was found in the prediction of liquid density for the methane-nbutane mixture $(7.5745 \%)$. The divergence in the fluid properties for the methane-nbutane mixture from the experimental could be attributed to the fact that the values of the compressibility factors and densities were not directly calculated from reported experimental data but were calculated using the Peng-Robinson equation of state. If the molar volumes had been measured and reported as in the other mixtures, the margins of errors might have been much smaller than the estimated values as shown in Table 7 .

Also, as can be seen across the scenarios investigated, the vapour phase properties are more accurately predicted than the liquid phase properties. This can be attributed to the inherent shortcomings with cubic equations of state which are known to give good descriptions of real fluid behaviour when applied to the gas phase, but lacks accuracy in the compressed 


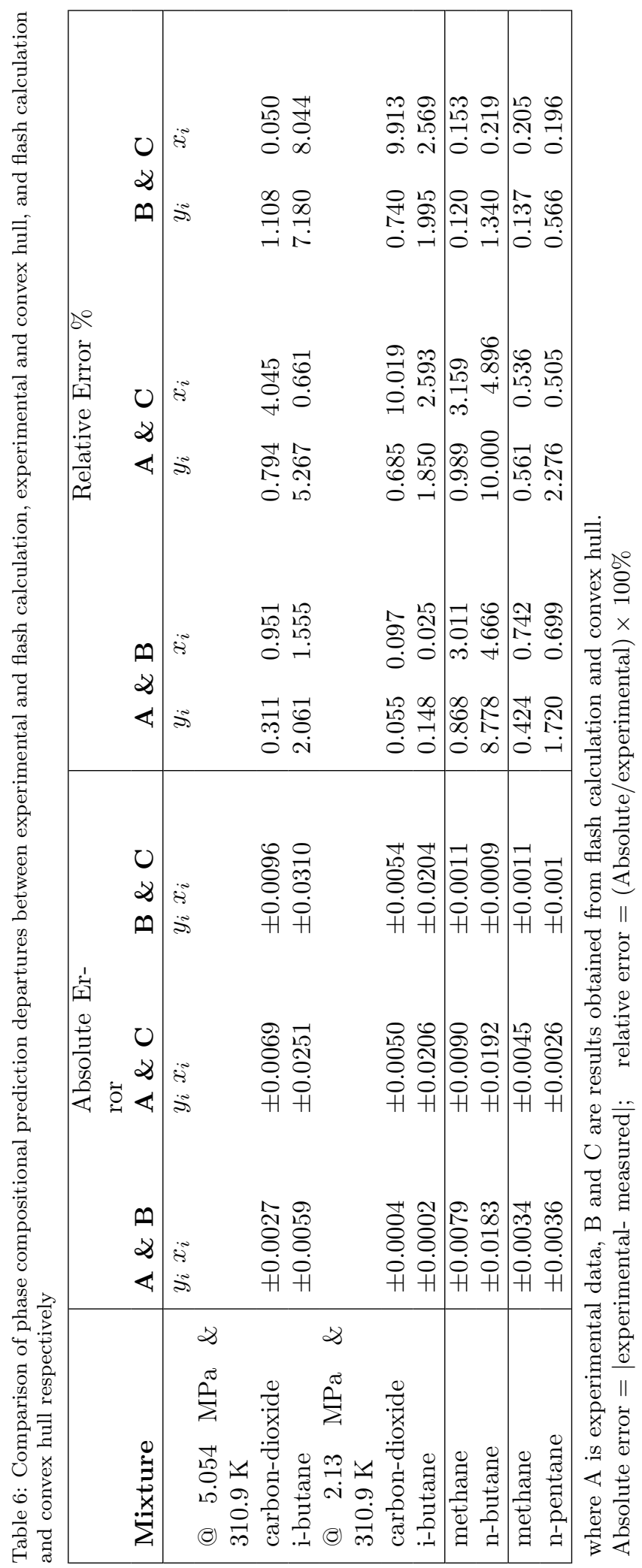




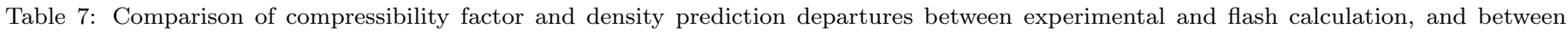
experimental and convex hull for different mixtures

\begin{tabular}{|c|c|c|c|c|}
\hline Fluid Properties & \multicolumn{2}{|c|}{ Absolute Error } & \multicolumn{2}{|c|}{ Relative Error \% } \\
\hline & flash cal & convex hull & flash cal & convex hull \\
\hline \multicolumn{5}{|l|}{$\mathrm{CO}_{2}-\mathrm{iC}_{4}$ mixture } \\
\hline$Z_{v}$ & 0.0408 & 0.0394 & 6.2233 & 6.0097 \\
\hline$Z_{l}$ & 0.0091 & 0.0057 & 5.6208 & 3.5207 \\
\hline$\rho_{v}$ & 9.4200 & 8.4200 & 6.8876 & 6.1564 \\
\hline$\rho_{l}$ & 30.3190 & 24.6080 & 5.0856 & 4.1276 \\
\hline @ $2.13 \mathrm{MPa} \& 310.9 \mathrm{~K}$ & & & & \\
\hline$Z_{v}$ & 0.0317 & 0.0313 & 3.7246 & 3.6776 \\
\hline$Z_{l}$ & 0.0023 & 0.0031 & 2.8152 & 3.6776 \\
\hline$\rho_{v}$ & 1.8700 & 1.8230 & 4.0386 & 3.9371 \\
\hline$\rho_{l}$ & 16.4713 & 18.9193 & 2.9574 & 3.0379 \\
\hline \multicolumn{5}{|l|}{$\mathrm{CH}_{4}-\mathrm{nC}_{4}$ mixture } \\
\hline$Z_{v}$ & 0.0167 & 0.0146 & 2.5380 & 2.2188 \\
\hline$Z_{l}$ & 0.0176 & 0.0179 & 4.6672 & 4.7468 \\
\hline$\rho_{v}$ & 1.2632 & 0.4835 & 0.8770 & 0.3357 \\
\hline$\rho_{l}$ & 30.3695 & 31.2491 & 7.3617 & 7.5745 \\
\hline \multicolumn{5}{|l|}{$\mathrm{CH}_{4}-\mathrm{nC}_{5}$ mixture } \\
\hline$Z_{v}$ & 0.0091 & 0.0073 & 1.2039 & 0.9657 \\
\hline$Z_{l}$ & 0.0018 & 0.0020 & 0.3809 & 0.4232 \\
\hline$\rho_{v}$ & 0.7567 & 0.0624 & 0.5119 & 0.0422 \\
\hline$\rho_{l}$ & 0.3089 & 0.3254 & 0.0789 & 0.0831 \\
\hline
\end{tabular}

liquid region where a wide density range is expected $[35,36]$. For the four scenarios investigated, the convex hull method did better in predicting the vapour compressibility factor and density than the flash calculation method. However, the flash calculation method did slightly better than the convex hull in predicting the liquid compressibility factor and density except in carbon-dioxide-isobutane mixture at $5.054 \mathrm{MPa}$ and $310.9 \mathrm{~K}$ where the convex hull is superior. With these small margins of errors between the conventional flash calculation and the convex hull methods in estimating VLE phase properties, it would be worth taking advantage of the time benefits associated with the convex hull method in calculating phase equilibria during compositional simulations.

\subsection{Tenary phase diagrams}

Ternary plots are prepared for mixtures of three pure species (or components), and show the composition space where any point within the space represents a particular combination of the three components. A multi-component convex hull function was used to develop ternary phase diagrams for a mixture of methane, decane, and nbutane at $160{ }^{\circ} \mathrm{F}(344.26 \mathrm{~K})$, and 1000 psia (6.89 MPa), 2000 psia (13.79 MPa), 3000 psia (20.68 MPa) and 4000 psia (27.58 MPa). The results are shown, together with output using data from other researchers [37], in Figure 8.

Ternary plots are usually prepared using a regular grid, and each point on the grid represents a point in composition space. That approach has been used to prepare the plots shown in Figure 8. It is possible, however, to perturb the grid by adding a random number to the values of the mole fractions that define the grid. If the random number is sufficiently small the topology of the regular grid is retained, although the grid points are shifted very slightly. If, however, the perturbations are sufficiently large, the topology of the grid changes. Both of these approaches were explored to establish that, regardless of the extent to which the regular grid was distorted by the introduced perturbations, the underlying data presented remained accurate. The approach was then extended to include additional composition points, within a particular element (or elements) of the grid, and the results checked to ensure that accuracy had been maintained. 


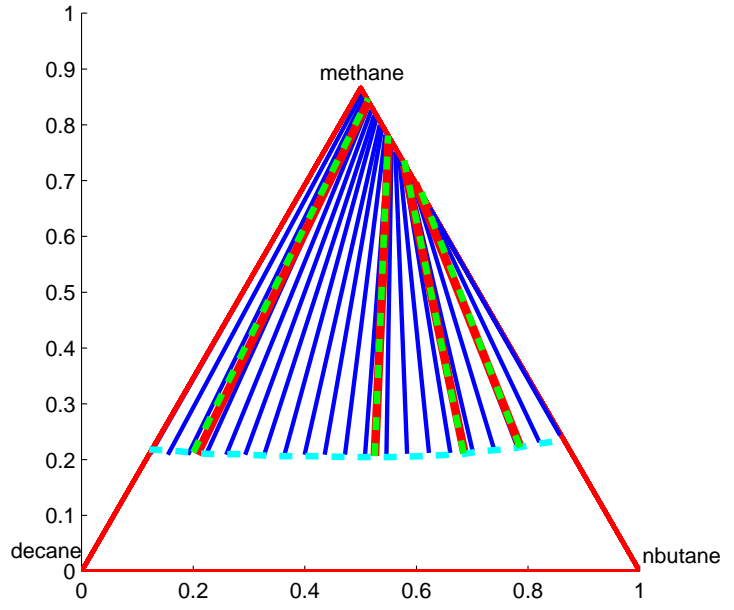

(a)

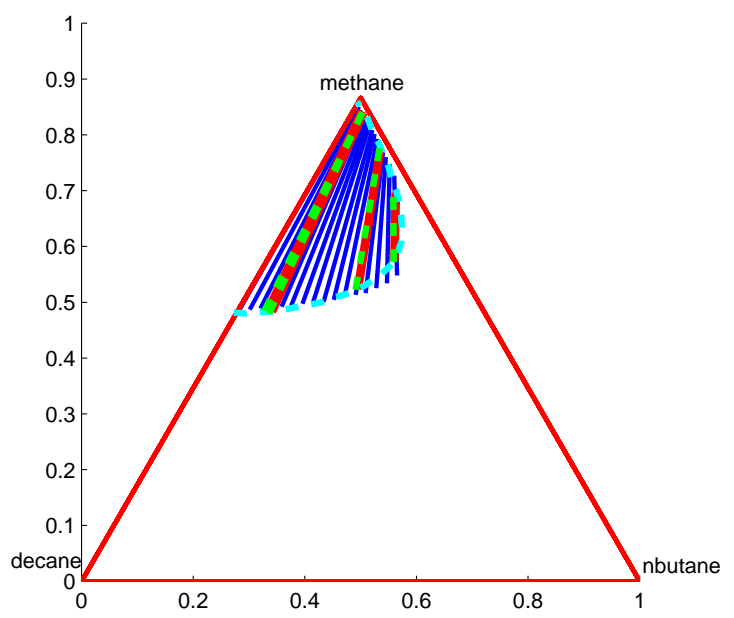

(c)

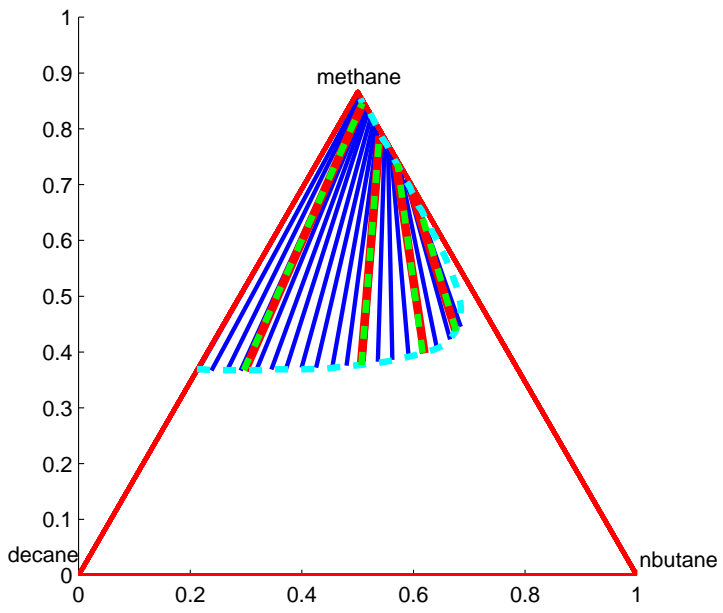

(b)

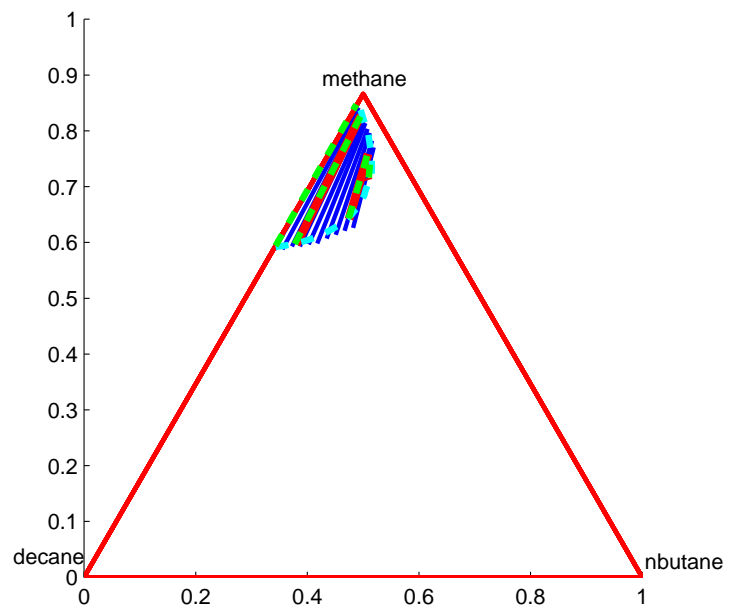

(d)

Figure 8: Ternary plots for methane-nbutane-decane mixture at $160^{\circ} \mathrm{F}(344.26 \mathrm{~K})$ for (a) $1000 \mathrm{psia}(6.89 \mathrm{MPa})$ (b) 2000 psia (13.79 MPa) (c) 3000 psia (20.68 MPa (d) 4000 psia (27.58 MPa).The ternary plot with the tie lines (also known as combining lines) shown in blue, were obtained using the approach presented here and the multi-component convex hull function. The cyan dashed curve represent the binodal curves for the different pressures. The red tie lines were plotted using data presented by Reamer et al. [37], the green dashed lines were obtained (by data capture) from figures presented on Petrowiki [38]. 
This permits a large number of points to be concentrated around the zone of a particular composition - a feature that is especially helpful when preparing a $p-T$ phase diagram for a specific composition as it greatly increases the accuracy that can be achieved close to the critical point.

\subsection{Pressure-temperature $(p-T)$ phase diagrams}

Pressure-temperature phase diagrams were also developed using this procedure for two and three components mixtures. These are shown in Figures 9 and 10. The procedure presented in this paper has the advantage that it can produce accurate data close to the critical point. For two component mixtures the process is relatively straightforward. Appropriate ranges

of pressure and temperature were selected and the program set to step through the ranges with a uniform step length. As the Gibbs can be accurately established for the specified composition, accurate 'hulls' were obtained for an appropriate range of temperatures and pressures.

In this fashion a matrix was constructed holding the mole fraction of the liquid phase $Q(i, j)$, for each temperature $T(i)$, and pressure $p(j)$, considered. Values of $Q$ in the single phase regions were easily identified and, for them, $Q(i, j)$ was set to a value greater than 1.0. A Delaunay triangulation was constructed with a colour scale to plot the phase diagram, ignoring values of quality greater than 1.0. By increasing the number of steps taken to progress through the range of temperatures and pressures considered, smoother plots were obtained. Two plots using different step lengths for a mixture of carbon dioxide and isobutane were produced and are shown in Figures 9(a) and 9(b). From this it can be seen that, even with a relatively small number of steps producing a very rough plot, the critical point can easily be identified (see Figure 9(a)). Figure 9(c) is a $p-T$ plot produced using published software available from mathworks [21], modified very slightly by the authors. The modifications include expressing the Peng-Robinson equation of state in terms of compressibility factor instead of molar volume and incorporating a code that plots pressure-temperature $p-T$, phase diagrams which is not available in the original code. This compares well with the plot shown in Figure 9(b). Figure 10 shows $p-T$ plots obtained using the procedure presented in this paper with a regular ternary grid refined only close to the composition (subplot (a)) compared with one produced using NIST Refprops [39] (subplot (b)).

\section{Conclusion and Further Discussion}

This paper presents a simple procedure for using a convex hull to calculate the vapour-liquid phase equilibria and develop phase diagrams for different mixtures. The convex hull is a non-iterative method that does not depend on the use of an accurate start value. With the convex hull method, mole fractions of the phases and other fluid phase properties at specific pressures and temperatures were easily determined. To increase confidence in the utility of this technique, it has been used to reproduce the results of Peng and Robinson's equation [19]; and to generate phase diagrams for binary and ternary mixtures of different components. The results from this procedure have been compared with published data, the flash calculation method and data obtained from a commercial software. The results shows good agreement, a demonstration of the reliability of this procedure.

Methods based upon the convex hull are non-iterative and hence have significant advantages over traditional RachfordRice iterative flash calculations, which can require a moderately accurate initial estimate in order to converge upon the solution. Without this limitation, convex hull methods can predict phase compositions, and close to the critical point, they are noticeably more accurate than the iterative methods. Significant performance improvements compared to the original Rachford-Rice have been obtained, and while there exist enhanced Rachford-Rice iterative calculations, the convex hull 


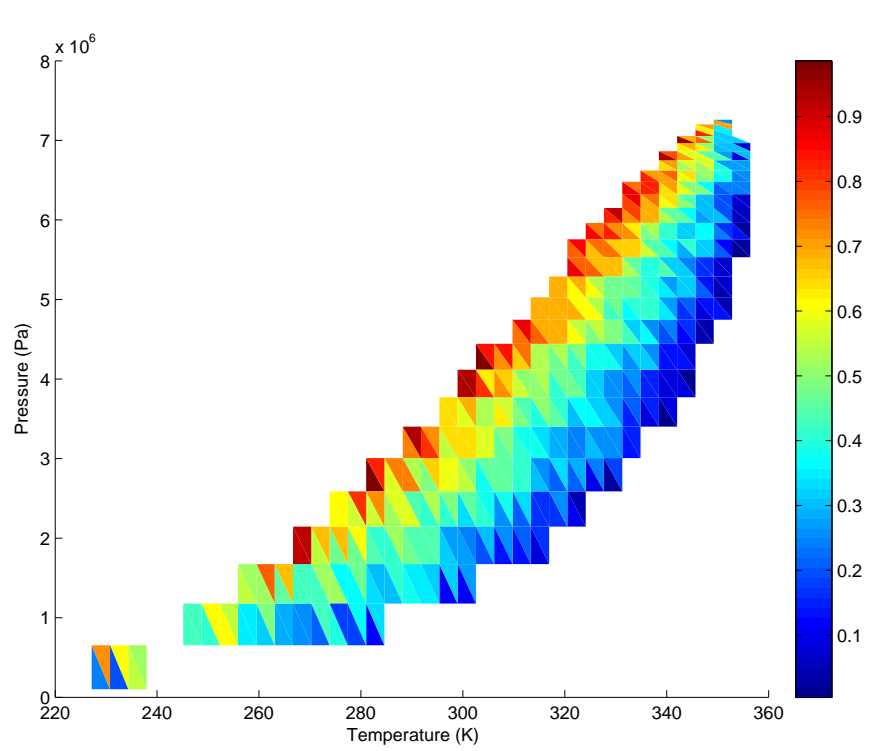

(a)

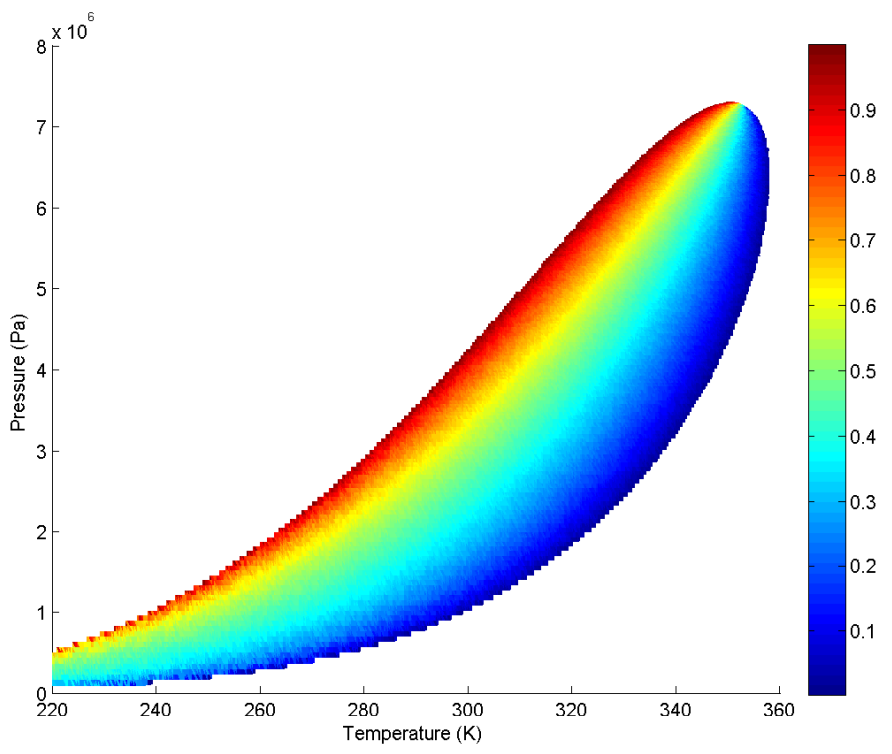

(b)

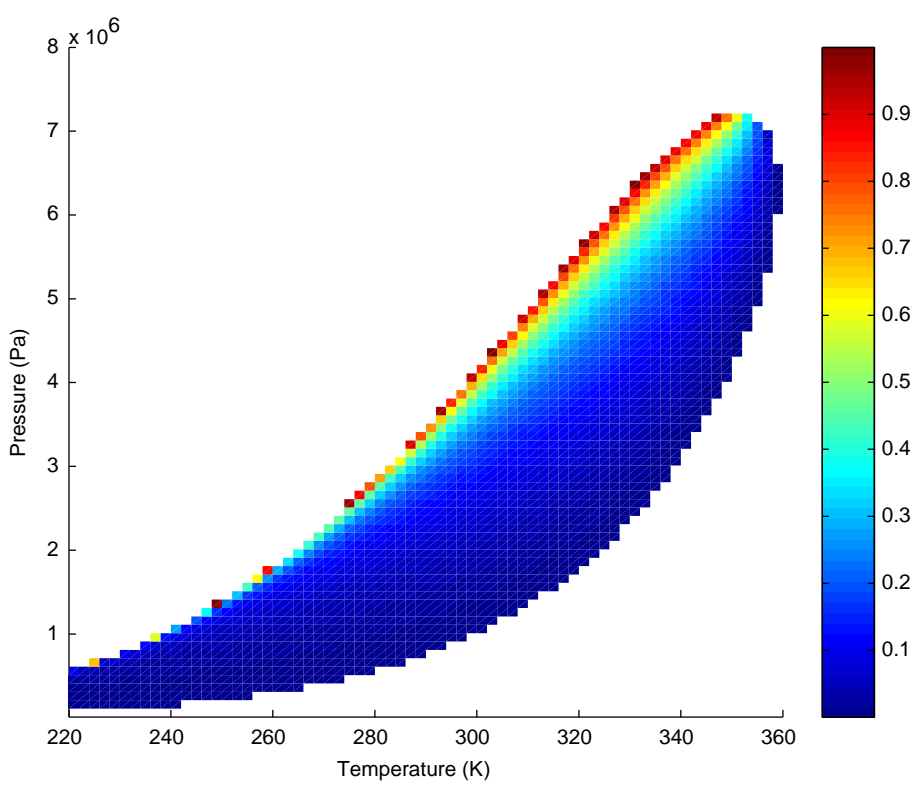

(c)

Figure 9: PT phase diagrams for 0.6 mole fraction Carbon Dioxide and 0.4 mole fraction of Isobutane. (a) With 40 steps; (b) with 320 steps and (c) developed using the modified code of Affrough [21] with 204 steps. The accurate treatment around the critical point even for few steps and long step lengths is clearly illustrated. 


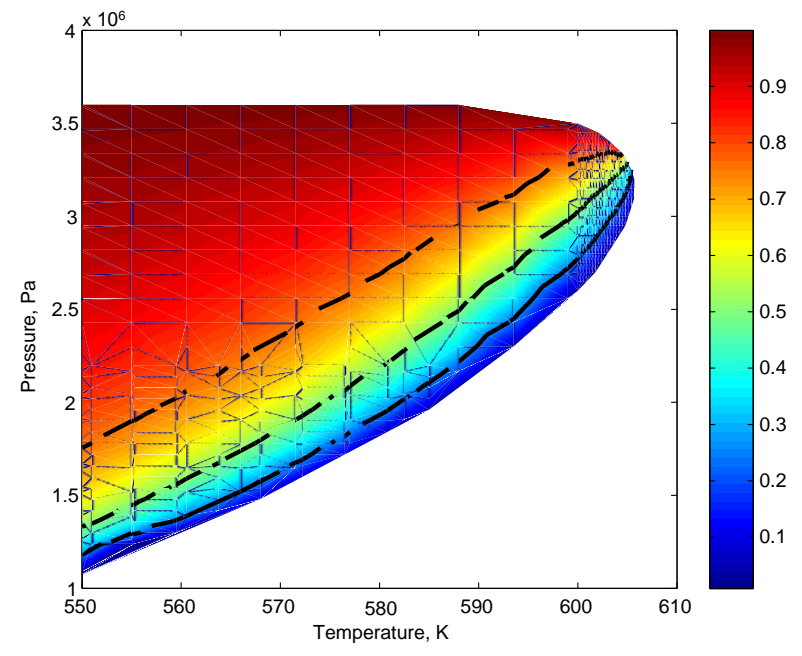

(a)

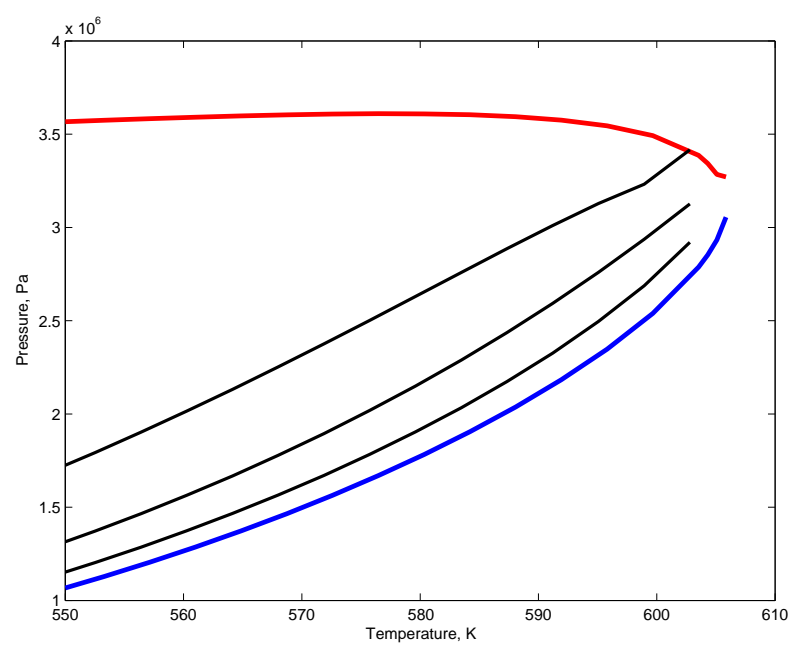

(b)

Figure 10: Pressure versus Temperature phase diagrams for 0.098 Methane, 0.101 nButane and 0.801 Decane mole fractions. (a) $p-T$ from convex hull using a basic grid with refinement close to the composition and also close to the critical point. The grid lines are shown in black. (b) Produced using a commercial software, RefProp. The fraction of the mass of the fluid that is present as liquid is indicted by the colour plot - red (1.0) is liquid and blue (0) is gas. The black lines indicate the quality at $0.25,0.5$ and 0.75 fraction of the mass of the fluid.

methods remain competitive. For example, the Li et al. [10] objective function is claimed to be $25 \%$ faster for threecomponent fluids and $40 \%$ faster for fifteen-component fluids than the Rachford-Rice method, and always converges at double precision accuracy no matter the overall composition and $K$-values. This was attributed to the fact that the objective function of Li et al. is less nonlinear than the Rachford-Rice objective function, and thus overcomes the problem of several iterations if the initial guess is not close to the correct root. Moreover, for these performance benefits to be achieved, the function of $\mathrm{Li}$ et al. [10] must be properly scaled near the critical points. However, with the convex hull method, no special scaling is required near the critical point, while it is also shown to be faster than the Rachford-Rice method by over $90 \%$ in all the cases investigated; an indication that if compared with the modified iterative models, it can still show improved performance.

Another modified Rachford-Rice function that is claimed to have performed better and faster than the conventional flash calculation method is that proposed by Gaganis and Varotsis [40]. In this model, a transformation of the RachfordRice function is made whereby it is written as a function of a single hyperbolic function instead of the vapour fraction. This function is monotonic and either strictly concave or convex, and so can be fitted by low rational model functions which do not require the calculation of a derivative, and thus the Newton-Raphson iteration. Instead a new algorithm is proposed to fit these rational model functions and determine the roots of these functions by performing simple computations which are used for the next estimation. Although this model is claimed to converge more rapidly than the conventional method, it is also iterative and requires an algorithm for fitting several rational model functions, before determining the roots, thereby increasing the complexity of flash calculations performed using this method. Moreover, the method also requires using a function to calculate properties at near-critical condition in each iteration. However, these complexities and model functions are not necessary for the convex hull method.

Although the approach presented in this paper is similar in some respects to some other tangent based methods [2, 41, $42,43]$, such methods require a high level of both thermodynamic and computational expertise. The method presented 
here uses an available, robust function whose implementation does not require such an advanced level of computational expertise. A multi-component convex hull function has been used here for three component mixtures and the procedure could be extended very easily to consider mixtures with more components. A procedure for increasing the accuracy of $p-T$ plots, particularly close to the critical point, by refining the ternary plots has also been presented and been shown to be beneficial. The reduced run-time mentioned in this paper is explored in greater detail and demonstrated by carrying out comparative analyses with the conventional flash calculations method.

\section{Acknowledgment}

Amieibibama Joseph wishes to thank Petroleum Technology Development Fund (PTDF) for their financial support which has made this research possible.

\section{Nomenclature}

\section{Symbol}

a

A

$B$

$b$

$b_{m}$

$f$

G

$H$

$M$

$n$

$p$

$R$

T

V

$x$

Z

\section{Greek symbols}

$\rho$

$\omega$

$\mu$

\section{Superscripts}

$v$

$l$

$\Theta, *$

\section{Units}

$\mathrm{m}^{6} \mathrm{~Pa} \mathrm{~mol}{ }^{-2}$

$-$

$\mathrm{m}^{3} \mathrm{~mol}^{-1}$

$\mathrm{m}^{3} \mathrm{~mol}^{-1}$

$\mathrm{Pa}$

$\mathrm{J}$

$\mathrm{J} \mathrm{mol}^{-1}$

$\mathrm{kg} \mathrm{mol}{ }^{-1}$

$-$

$\mathrm{Pa}$

$\mathrm{m}^{3} \mathrm{~Pa} \mathrm{~K} \mathrm{~K}^{-1} \mathrm{~mol}^{-1}$

$\mathrm{K}$

$\mathrm{m}^{3} \mathrm{~mol}^{-1}$

$-$

$-$

\section{Description}

Peng-Robinson EOS attractive parameter for mixture

Peng-Robinson EOS parameter

Peng-Robinson EOS parameter

Peng-Robinson co-volume parameter for the i-th component

Peng-Robinson co-volume parameter for mixture

fugacity

Gibbs energy

enthalpy

apparent molecular weight

number of moles

pressure

gas constant

temperature

molar volume

mole fraction

gas deviation factor

density

acentric factor

chemical potential

vapour phase

liquid phase

reference state

\section{Subscripts}



$i, j, k$
component index
$m$
mixture
$c$
critical property

\section{References}

[1] T. Ahmed. Vapor-Liquid Phase Equilibria Calculations. Gulf Professional Publishing, Massachusetts, USA, 2010.

[2] L. E. Baker, A. C. Pierce, and K. D. Luks. Gibbs energy analysis of phase equilibria. Soc. Petrol. Eng. J., (SPE9806-PA), 1982.

[3] F. M. Orr Jr and J. J. Taber. Phase Diagrams. In Petroleum Engineering Handbook, Chap. 23. Society of Petroleum Engineers, Richardson, Texas, USA, 1987.

[4] C.U. Ikoku. Natural Gas Production Engineering. Krieger Publishing Company, Malabar, Florida, USA, reprint edition, 1992.

[5] M. Kariznovi, H. Nourozieh, and J. Abedi. Vapour-liquid phase equilibria and physical properties measurements for ternary systems (methane + decane + hexadecane). J. Chem. Eng. Data, 57(9):2535-2542, 2012.

[6] M. L. M. L. Michelsen, W. Yan, and E. H. Stenby. A comparative study of reduced-variables-based flash and conventional flash. SPE J., 18(05):952 - 959, 2013.

[7] M. L. Litvak and C. H. Wang. Integrated reservoir and surface pipeline network compositional simulations. SPE48859-MS, presented at the SPE International Oil and Gas Conference and Exhibition, Beijing, China, 1998.

[8] H. H. Rachford Jr. and J.D. Rice. Procedure for use of electronic digital computers in calculating flash vaporization hydrocarbon equilibrium. J. Pet. Tech, 4(10):327-328, 1952.

[9] M. Heidari, L. X. Ngheim, and B. B. Maini. Improved Isenthalpic Multiphase Flash Calculations for Thermal Compositional Simulators. SPE-170029-MS, presented at the SPE Heavy Oil Conference Canada, Alberta, Canada, 2014 .

[10] Y. Li and R. T. Russell. A Rapid and Robust Method To Replace Rachford-Rice in Flash Calculations. SPE-106080, presented at the SPE Reservoir Simulation Symposium, Houston, Texas, USA, 2007.

[11] H. Pan and A. Firoozabadi. Fast and Robust Algorithm for Compositional Modelling: Part II - Two-Phase Flash. SPE J., 7(1):79-89, 2003.

[12] A. Firoozabadi and H. Pan. Fast and Robust Algorithm for Compositional Modelling: Part I - Stability Analysis Testing. SPE J., 7(1):79-89, 2002.

[13] D. V. Nichita and F. Minescu. Efficient phase equilibrium calculation in a reduced flash context. Can. J. Chem. Eng., 82:1225-1238, 2004.

[14] C. P. Rasmussen, K. Krejbjerg, M. L. Michelsen, and K. E. Bjurstrøm. Increasing the computational speed of flash calculations with applications for compositional, transient simulations. SPE Res. Eval. Eng., 9(01):32-38, 2006. 
[15] M. L. Michelsen and J. Mullerup. Thermodynamic Models: Fundamentals and Computational Aspects,. Tie-line Publications, Holte, Denmark, second edition, 2007.

[16] D. V. Voskov and H. A. Tchelepi. Compositional space parametrization for miscible displacement simulation. Trans. Porous Media, 75(1):111-128, 2008.

[17] D. V. Voskov and H. A. Tchelepi. Tie-simplex based mathematical framework for thermodynamical equilibrium computation of mixtures with an arbitrary number of phases. Fluid Phase Equilibr., 283(1-2):1-11, 2009.

[18] W. Yan, A. Belkadi, E. H. Stenby, and M. Michelsen. Study on the application of the tie-line-table-look-up-based methods to flash calculations in compositional simulations. SPE J., 18(05):932-942, 2013.

[19] D. Y. Peng and D. B. Robinson. A new two-constant equation of state. Ind. Eng. Chem. Fundamen., 15(1):59-64, 1976.

[20] Sigurd Skogestad. Chemical and energy process engineering. CRC Press (Taylor \& Francis Group), 2008.

[21] A. Affrough. Vapor Liquid Equilibrium Calculations (flash) using Peng-Robinson Equation of State. Available at http://www.mathworks.com/matlabcentral/fileexchange/48836-flashcode/content/Flashcode/flashcode.m, 2014. Accessed 20 May, 2015.

[22] B. Barber, D.P. Dobkin, and H. HuhdanPAA. The quickhull algorithm for convex hulls. ACM Trans. Math. Software, 22(4):469-483, 1996.

[23] A. Mitsos and P.I. Barton. A dual extremum principle in thermodynamics. AIChE, 53(8):2131-2147, 2007.

[24] J. Boardman. Automating spectral unmixing of aviris data using convex geometry concepts. 4th JPL Airborne Geoscience Workshop (Washington, D.C), JPL, Pasadena, California, 1993.

[25] J. Weeks. Convex hulls and isometrics of cusped hyperbolic 3-manifolds. Technical report, Tech. Rep. TR GCG32, The Geometry Centre, University of Minnesota, Minneapolis, USA, 1991.

[26] O. Ryll, S. Blagov, and H. Hasse. Convex envelope method for the determination of fluid phase diagrams. Fluid Phase Equilibr., 324(0):108 - 116, 2012.

[27] I. Müller and W. H. Müller. Fundamentals of Thermodynamics and application. Springer-Verlag, Berlin Heidelberg, 2009.

[28] A. Péneloux, E. Rauzy, and R. Fréze. A consistent correction for Redlich-Kwong-Soave volumes. Fluid Phase Equilibr., 8(1):7-23, 1982.

[29] Z. Nasri and H. Binous. Application of the Peng-Robinson equation of state using Matlab. Chem. Eng. Edu., $43(2): 1-10,2009$.

[30] Z. Xu and S. I. Sandler. Application to mixtures of the Peng-Robinson equation of state with fluid-specific parameters. Ind. Eng. Chem. Res., 26(6):1234-1238, 1987. 
[31] V. M. Berry and B. H. Sage. Phase behavior in binary and multicomponent systems at elevated pressures-pentane and methane-n-pentane. National Standard Reference Data Series (NSRDS-NBS) 32, National Bureau of Standards, 1970.

[32] D. G. Elliot, R. J. J. Chen, P. S. Chappelear, and R. Kobayashi. Vapor-liquid equilibrium of methane-butane system at low temperature and high pressures. J. Chem. Eng. Data, 19(1):71-77, 1974.

[33] J. C. Tsai and Y. P. Chen. Application of a volume-translated Peng-Robinson equation of state on vapor-liquid equilibrium calculations. Fluid Phase Equilibr., 145(2):193-215, 1998.

[34] G. J. Besserer and D.B. Robinson. Equilibrium-phase properties of isobutane-carbon dioxide system. J. Chem. and Eng. Data, 18(3):298-301, 1973.

[35] K. H. Kumar and K. E. Starling. The most general density-cubic equation of state: application to pure nonpolar fluids. Ind. Eng. Chem. Fund., 21(3):255-262, 1982.

[36] B. E. Poling, E. A. Grens, and J. M. Prausnitz. Thermodynamic properties from a cubic equation of state: avoiding trivial roots and spurious derivatives. Ind. Eng. Chem. Process Des. Dev., 20(1):127-130, 1981.

[37] H. H. Reamer, J. M. Fiskin, and B. H. Sage. Phase equilibria in hydrocarbon systems. Ind. Eng. Chem., 41(12):2871$2875,1949$.

[38] Ternary Phase Diagrams, http://petrowiki.org/ternary_phase_diagrams. Accessed 12 December, 2015.

[39] E. W. Lemmon, M. L. Huber, and M. O. Mclinden. Reference fluid thermodynamic and transport propertiesREFPROP. Standard reference database 23, version 9.0, thermodynamic property division, NIST, 2010.

[40] V. Gaganis and N. Varotsis. A New Transformation for the Rapid Solution of the Rachford-Rice Equation in Phase Split Calculations. SPE-150932-MS, presented at the North Africa Technical Conference and Exhibition, 20-22 February, Cairo, Egypt, 2012.

[41] M. L. Michelsen. The isothermal flash problem. Part II. Phase-split calculation. Fluid Phase Equilibr., 9:21-40, 1982.

[42] M. L. Michelsen. The isothermal flash problem. Part I. Stability. Fluid Phase Equilibr., 9:1-19, 1982.

[43] L. Qiu, Y. Wang, Q. Jiao, H. Wang, and R. D. Reitz. Development of a thermodynamically consistent, robust and efficient phase equilibrium solver and its validations. Fuel, 115:1-16, 2014. 